\title{
A Reference Map of Microbiome Composition in Crop Production Across 8 Chinese Provinces
}

Haiyang Hu ( $\square$ huhaiyang@sjtu.edu.cn )

Shanghai Jiao Tong University https://orcid.org/0000-0002-2059-5115

Yunli Liu

Shanghai Jiao Tong University

Zhaoyong Xu

Shanghai Jiao Tong University

Yiqun Huang

Shanghai Jiao Tong University

Hongzhi Tang

Shanghai Jiao Tong University

\section{Research article}

Keywords: Crop microbiota, leaves, Core bacterial community, industrial factors

Posted Date: November 23rd, 2021

DOI: https://doi.org/10.21203/rs.3.rs-1081017/v1

License: (c) (1) This work is licensed under a Creative Commons Attribution 4.0 International License. Read Full License 


\section{Abstract}

Background: Although microbiome profiling of crop leaves is a relatively new research area, we know that leaf microorganism communities affect the leaf chemical composition, promote growth, and confer protection against deleterious microbes.

Results: Here, we used 16S rDNA sequencing to profile the microbiomes of 78 primary dried tobacco leaf samples from plants grown at 26 locations in 8 Chinese provinces. After analyzing leaf microbial communities and identifying 4,473 operational taxonomic units (OTU) representing 1,234 species, we obtained a national core microbiome with 14 OTU representing 9 species, with functions for processes including nitrogen fixation, detoxification of diverse pollutants, and heavy-metal reduction. Although there are no obvious impacts from single environmental factors (e.g., temperature, precipitation), there are significant differences in microbial community structures among provinces and clear clustering by region. Finally, LEfSe analysis screened the significantly different species between different sampling locations.

Conclusions: Our study demonstrates that both climactic and industrial factors drive microbial diversity on crop plant leaves, and many potentially useful functional bacterial resources present on tobacco leaves are still waiting for harnessing for a variety of bioprocess, agricultural, and environmental detoxification applications.

\section{Background}

With the help of recently developed techniques for culture-independent microbial identification, the microbial diversity and distribution of various environments can now be readily studied, including the human gut, wastewater treatment plants, and soils [1-3]. Such studies are helpful for improving our understanding of bioprocesses occurring in these environments and our understanding of risks to human health, and these studies also facilitate harnessing of microorganisms for human use [4]. Of particular note, both Jizhong Zhou and Noah Fierer have recently organized global-sampling projects, respectively collecting 1200 activated sludge samples from 23 countries on six continents and 237 soil samples from six continents $[5,6]$. These studies yielded "most wanted" lists for future experimental efforts to understand microbiome of activated sludge and soil, and it is clear that such efforts can greatly promote microbial resource development in related fields.

Studies of multiple crop species have shown that microorganism's resident on the surface of plant leaves (e.g., Populus trichocarpa, Arabidopsis, Nicotiana tabacum L.) alter the chemical composition of leaves, promote crop growth, and confer antibacterial effects [7-10]. Nevertheless, leaf profiling of crop species is a relatively new research area, so there are many gaps in our knowledge, including about the variations in the basic microorganism compositions and about the factors which can influence the function of particular taxa on leaves. Bacillus subtilis and Bacillus cricosporum were isolated from fermented leaves 
in 1967, both of which release pleasant fragrance compounds [11], and Enterobacter cloacae was isolated from the surface of tobacco leaves in 1983 and showed that it degrades nicotine [12].

More recently, Mingqin Zhao successfully utilized a mixture of four dominant bacteria alongside $a$ amylase and protease treatments during leave fermentation to reduce irritant gas production $[13,14]$. Advancing this research area beyond screening microorganisms using conventional media culture, Jingwen Huang and Can Su applied 16S rDNA sequencing to analyze the bacterial communities of unaged and aging flue-cured tobacco of the 'K326' and 'Zimbabwe' cultivars $[15,16]$. There have also been studies of the microbial diversity of tobacco in China, including analysis of samples from Fujian Province [17-19]. These studies have discovered some new microorganisms, including Sphingomonas, Enterobacter, and Pantoea, among others, which could not have been detected using traditional culturebased methods.

In the present study, we profiled the microbiomes of 78 primary dried tobacco leaf samples from plants grown at 26 locations in 8 Chinese provinces. We systematically analyzed the leaf microbial community across the country and obtained a "core microbiome" for the whole country and core microbiomes for each of the 8 provinces. We also analyzed the predicted functions of the OTUs in the core microbiomes, which highlighted crop-growth promotion and detoxification of diverse pesticides and heavy metals. There were no obvious impacts from single environmental factors (temperature, precipitation, altitude, longitude, or latitude) on shaping microbial diversity, but the samples did tend to cluster together by province. Further analysis supported that leaf microbiomes are significantly affected by locally specific factors including both climactic and industrial factors.

\section{Methods}

\section{Sample collection}

Crop leaves were collected from 26 locations in China. A total of 78 samples were collected from 26 sampling locations in China. We collected at least 3 samples in each location, and collected more than 3 samples per site in 16 locations. Crop samples were stored at $4{ }^{\circ} \mathrm{C}$ and handled within a month.

\section{Sample treatment}

A whole crop leaf from the collected sample was settled in a sterilized flask with $300 \mathrm{~mL}$ sterilized $1 \mathrm{M}$ phosphate buffer $\left(\mathrm{pH}\right.$ 7.0). The flask was further shaked at $16^{\circ} \mathrm{C}$ for $30 \mathrm{~min}$. The supernatant was filtered with four layers of sterilized gauzes to remove residue, and then re-filtered with $0.22 \mu \mathrm{m}$ filter membrane to collect microorganisms. The membranes carrying gathered microorganisms were transfer to a sterile centrifugal tube, frozen in liquid nitrogen for $15 \mathrm{~min}$, and finally stored at $-80{ }^{\circ} \mathrm{C}$.

\section{S rDNA gene sequencing and sequence processing}

The samples were sent to Shanghai Personal Biotechnology Co., Ltd for DNA extraction and further sequencing. The primers 27F (5'-AGAGTTTGATCMTGGCTCAG-3') and 1492R (5'-ACCTTGTTACGACTT 
$\left.-3^{\prime}\right)$ were applied to amplify 16 S rDNA.

Pacbio Sequel platform (the third-generation sequencing platform) was applied to sequence the fulllength 16S rDNA of the community. To ensure the reliability and accuracy of the analysis results, QIIME software (Quantitative Insights Into Microbial Ecology, v1.8.0, http://qiime.org/) was used to exclude uncertain sequences. Sequences with $5^{\prime}$ mismatched base number $>5$ and with continuous same base number>8 were removed. Then we used USEARCH (v5.2.236, http://www.drive5.com/usearch/) to check and remove chimera sequence and obtain 945,434 processed sequences.

\section{Sequence comparison against reference databases}

UCLUST, a sequence alignment tool, was used to merge the 945,434 processed sequences and demarcate OTUs according to $97 \%$ sequence similarity. The sequences with the highest abundance in each OTU were selected as the representative sequences of this OTU. The representative sequences of OTU were compared with the NCBI database to obtain the taxonomic information.

\section{Species abundance distribution (SAD) fitting}

We used different models to predict SAD of each sample, such as Poisson lognormal, log-series, Brokenstick and Zipf models, which could be beneficial for deduce the percentages of variations in abundance among species by comparing the observed and predicted SADs.

\section{Core bacterial community determination}

OTUs meeting all three criteria were defined as core bacterial community. First, the mean relative abundance of core OTUs must more than $1 \%$ in every sample. Second, core OTUs should be identified in more than $80 \%$ of all samples. Third, the OTUs were identified as abundant when they made up the top $80 \%$ of the reads in the sample. The core OTUs should be identified as abundant in at least half of all the samples.

\section{a-diversity}

a-diversity indices, including Shannon [20], Simpson [21], Chao1 [22] and ACE [23], were calculated using QIIME software. Chao1 and ACE indices were employed to determine community richness, and Shannon and Simpson indices were used to determine community diversity. Then we used QIIME and R software to draw the rarefaction curve and species accumulation curve respectively. By randomly extracting a certain number of sequences from each sample, rarefaction curve is able to predict the total number of species and relative abundance of each species in the sample at a given sequencing depth. Species accumulation curve is able to judge whether the sample numbers were sufficient or not and estimate the community richness.

\section{B-diversity}


The weighted and unweighted UniFrac distances were calculated to represent the phylogenetic $\beta$-diversity using the R software. With QIIME software, the UPGMA clustering analysis were performed based on weighted and unweighted UniFrac distances respectively. R software was used to draw PCoA and NMDS diagrams respectively which could show the differences between different samples.

\section{Redundancy analysis (RDA)}

RDA analysis was a typical constrained sequencing method. Multiple linear regression was used to fit the data with several given influencing factors including longitude, latitude, altitude, temperature and precipitation (Table S5) test was used to determine whether the factor had significant influence on the bacterial composition or not. We identify "significant difference", if the value of -log10 $(P)$ is over 1.3.

\section{Construction of phylogenetic tree and taxonomic tree}

A phylogenetic tree with OTU representative sequences were constructed by Fast Tree tool. MEGAN (http://www.megasoftware.net/) was used to map the abundance information and taxonomy composition data of OTU contained in each sample to the taxonomic tree provided by NCBI Taxonomy (https://www.ncbi.nlm.nih.gov/taxonomy) so that in a standard classification system, the specific composition of all samples at each classification level was presented uniformly.

\section{LEfSe analysis}

LEfSe is an analysis method based on linear discriminant analysis (LDA). With the Galaxy online platform (http://huttenhower.sph.harvard.edu/galaxy/), the relative abundance of the species-level matrix was analyzed. LEfSe gives the logarithmic score value of LDA $>2$, and $P$ value $<0.05$.

\section{Functional prediction with PICRUSt}

Based on the measured 16S rDNA full-length sequences of microorganisms, the gene functional spectrum of common ancestor genes was deduced. By inferring unmeasured 16S rDNA full-length sequences of Greengenes database, the gene functional prediction spectrum of the complete spectrum of archaea and bacteria was constructed. These two kinds of 16S rDNA gene sequences were compared and the "nearest neighbor of the reference sequence" of each sequence was found. According to the rRNA gene copy number of the "nearest neighbor of reference sequence", the obtained OTU abundance matrix was corrected. The data of bacterial composition was "mapped" to the gene functional spectrum database of KEGG, and the metabolic function of bacterial community was predicted.

\section{Determination of metals-Inductively coupled plasma mass spectrometry (ICP-MS)}

Preparation for tobacco digestion: Crushed the tobacco leaves and dried at $60{ }^{\circ} \mathrm{C}$ to constant weight. Then weighed $0.2 \mathrm{~g}$ tobacco powder into a shake flask and added $10 \mathrm{~mL}$ mixed acid $\left(\mathrm{HNO}: \mathrm{HClO}_{4}=4: 1\right)$ overnight (12h). The solution was digested by electrothermal digestion until the solution was transparent. Then $1 \mathrm{~mL} 1 \% \mathrm{HNO}_{3}(\mathrm{~V}: \mathrm{V})$ was added to diluted to $25 \mathrm{~mL}$. The treated samples were detected by ICP-MS. 


\section{Results}

We obtained a total of 945,434 sequences from 78 crop samples at 26 sampling locations distributed across 8 Chinese provinces (Fig. 1a). Operational taxonomy units (OTUs) were demarcated according to $97 \%$ sequence similarity. Finally, a total of 4,473 OTUs were identified, and these were classified into 11 phyla, 26 classes, 69 orders, 151 families, 440 genera, and 1234 species. We detected 1399 OTUs per province on average, with a minimum of 620 OTUs for Chongqing (CQ) and a maximum of 2473 OTUs in Yunnan (YN) (Fig. 1b).

The classification tree for all samples visualized by GraPhIAn is shown in Fig. 1c, as well as the relative abundances of the microorganisms at the genus level is shown in Fig. 1d (Fig. S1 shows the community compositions at different taxonomic levels). A total of 440 genera were identified from the 78 samples, among which the top 13 genera (in terms of relative abundance) accounted for $82.09 \%$ of all OTUs for the entire dataset (details in Table S1). Notable genera from among these top 13 included Salmonella (4.3\%), which is harmful for human health.

\section{Species abundance distribution (SAD)}

Species abundance distribution is a description of the abundance (number of individuals observed) for each different species encountered within a community; this can also be described as a community-level metric denoted by $\Phi(n)$ that tells us the probability of a given species having " $n$ " abundance [24]. Thus, $S A D$ is one of the major tools in ecology. In the present study, we applied common SAD models, including Poisson lognormal, log-series, Broken-stick, and Zipf to predict species abundance distributions of crop leaf bacterial communities. The Poisson lognormal model had the best fit for the SAD among all samples, and was able to explain $95.34 \%$ of the variation of the crop leaf bacterial SAD, thus outperforming the $84.77 \%$ for lognormal, $78.88 \%$ for log-series, $84.11 \%$ for Zipf, and $10.23 \%$ for the Broken-stick model.

\section{Functional prediction with PICRUSt}

We employed phylogenetic investigation of communities by reconstruction of unobserved states (PICRUSt) [25] to explore the potential functions of the microorganisms detected in our samples. The abundance distributions showed between $43.10 \%-50.48 \%$ of the predicted pathways from the OTU data were of the metabolism type (Fig. S2). In more detail, the values of the relative abundance for pathways including "carbohydrate metabolism", "amino acid metabolism", "energy metabolism", "metabolism of cofactors and vitamins", "xenobiotics biodegradation" and "metabolism, nucleotide metabolism, and lipid metabolism" were $8.91 \%-10.631 \%, 8.33 \%-10.27 \%, 4.60 \%-9.11 \%, 3.69 \%-5.48 \%, 2.13 \%-3.90 \%, 2.75 \%-$ $3.19 \%$ and $2.71 \%-3.62 \%$, respectively (Fig. S2).

\section{a-Diversity analysis of the crop samples}

We next examined microbial diversity and community composition by characterizing $a$-diversity. First, we confirmed that both the Shannon rarefaction curve and the species accumulation curve indicated 
sufficient sequencing depth to informatively reveal the community diversity of the samples (Fig. 2a, 2b). The $a$-diversity indices, including Shannon, Simpson, Chao1, and ACE, were applied to assess the microbial diversity of the samples. In the national scale, the Chao1 and ACE index values were quite high (Table S2), underscoring the high bacterial richness among the samples. Nevertheless, the values of the Shannon and Simpson index were quite low (Table S2), suggesting that the relative abundances of the taxa in a given sample differed greatly with other samples. The box plot of Shannon and Simpson index indicated that microbial diversity was highest in Fujian province and lowest in Henan province (Fig. 2c, 2d).

\section{A national core bacterial community}

We next used standard criteria for defining community selection to characterize the core bacterial communities at a country-wide scale (i.e., analysis of the samples from 8 provinces) based on abundance and frequency data (see Methods for details) [26,27]. This type of analysis can identify the dominant species in each of the in situ environments represented by the 26 sampling sites. To determine the core community, OTUs were defined as abundant when they made up the top $80 \%$ of the reads in a sample (when ranked by decreasing OTU abundance) (Fig. 3a). When the cumulative read abundance reached $80 \%, 245$ OTUs were observed. The frequency of each OTU was also considered in the core community selection. There are 24 OTUs present in more than $80 \%$ of the samples, and these together comprise $85.4 \%$ of the relative abundance for the whole data set (Fig. 3b). Following principles for core community selection, we cataloged a national core bacterial community, which included $0.31 \%$ of the OTUs (14 OTUs) yet occupied $31.01 \%$ ( $\pm 3.94 \%)$ of the total relative abundance of the whole data set comprising all 78 samples (Fig. 3c, 3d, Table 1). The national core community consisted of the following genera: Mastigocoleus (9.35\% $\pm 6.89 \%)$, Pseudomonas (4.51\% $\pm 2.43 \%)$, Salmonella $(3.27 \% \pm 6.24 \%)$, Leclercia $(3.31 \% \pm 2.81 \%)$, Methylobacterium $(2.46 \% \pm 1.69 \%)$, Enterobacter $(2.17 \% \pm 2.48 \%)$, Atlantibacter $(2.14 \% \pm 3.77 \%)$, Pantoea $(1.93 \% \pm 2.66 \%)$, and Sphingomonas $(1.88 \% \pm 1.57 \%)$.

Among these of the national core bacterial community, Salmonella enterica is a well-studied pathogenic bacterium, and is present in almost all of the samples. The function of Methylobacterium goesingense, Sphingomonas roseiflava and Sphingomonas aurantiaca are unclear. Mastigocoleus testarum is a kind of cyanobacteria previously implicated in biological erosion [28], and Atlantibacter hermannii was reclassified from Escherichia hermannii and Salmonella subterranean [29], but there is little other information available. The remaining 4 genera-Pseudomonas (Pseudomonas oryzihabitans, Pseudomonas straminea), Leclercia (Leclercia adecarboxylata), Enterobacter (Enterobacter soli), and Pantoea (Pantoea agglomerans) - have been implicated to function in processes including nitrogen fixation [30], heavy metal reduction [31], insecticidal activity [32,33] and degradation of PAHs [34], lignin [35], and indole-3-acetic acid [36] (Table 1).

\section{Provincial core bacterial communities}

The core bacterial communities for each province were then determined using the same method as for the national core bacterial community (Table S3), and we prepared lists for beneficial bacteria that are 
specific to each province (Table S4). For example, in Yunnan province, OTU_4397 was close to Ochrobactrum anthropi, which is related to aniline degradation [37], and heavy metal detoxification [38]. Ochrobactrum lupine (OTU_8084) was core community member in Sichuan and Chongqing, and has been shown to degrade pesticides including chlorothalonil, beta-cypermethrin, and 3-phenoxybenzoic acid [39, 40]. Ochrobactrum pseudintermedium (OTU_8922), specific to Fujian, is known to degrade PAHs [41]. Moreover, Acinetobacter sp. (OTU_12502, OTU_9277, OTU_11315) was among the core bacterial communities of Guizhou, Fujian, Hunan, Chongqing, and Sichuan, and have been shown to degrade PAHs and nicotine and to detoxify heavy metals [42]. A recently reported cellulose-degrading specy, Beijerinckia fluminensis (OTU_2253, OTU_13065), was found in the core bacterial communities of both Fujian and Heilongjiang Province [43].

We also identified pathogenic bacteria among the province core communities. For instance, the black rot causal pathogen Xanthomonas campestris was in the Yunnan core [44]. We also found bacteria with reported links to human diseases, including Kluyvera intermedia [45] in the Chongqing core,

Stenotrophomonas maltophilia [46] in the Heilongjiang core, Bordetella petrii and Bordetella hinzii [47, 48] in the Fujian core. In terms of both diversity and abundance of pathogenic bacteria, the Chongqing core community had the largest number (3 species, 8.85\%).

\section{$\beta$-Diversity analysis}

We also conducted $\beta$-diversity analysis to characterize relationships among the samples. Two types of clustering analysis-PcoA analysis of unweighted Unifrac distance and UPGMA (unweighted pair-group method with arithmetic means) -both showed that the samples tend to cluster together by province, suggesting a significant effect from sampling location on microbial community composition (Fig. S3). A multi-response permutation procedure was applied to determine the composition difference between each province. More than half of the groups showed significant differences, again indicating that the microbial community composition varies greatly from province to province (Fig. 4a).

\section{Redundancy analysis (RDA) and correlation with environmental factors}

We employed RDA to explain the composition difference and to determine the influences of local environmental factors on microbial community composition, including temperature, precipitation, altitude, longitude, and latitude (Table S5). The microbial community composition of the samples in Yunnan province was most strongly affected by altitude, whereas that of Henan province was most strongly affected by latitude. However, none of the single environmental factors showed a significant effect on microbial communities at the national scale (Fig. 4b). Latitudinal-diversity gradients for all samples were also analyzed, but no obvious influence was detected for latitude or longitude, which is consistent with previous reports of microbial latitudinal diversity gradients [49] (Fig. S4).

\section{Locally specific factors drive community compositions}


Our data support that the microbial communities are obviously affected by local environments, but did not exhibit obvious relationships to single ecological factors. Thus, we screened the significantly different species between different sampling locations with LEfSe (Linear discriminant analysis effect size) analysis attempting to unravel key factors influencing microbial community structure. This identified 8 species in Mangdui, Menglijiaojidi, Qujing, and Chuxiong of Yunnan province, Xuchang and Luoyang of Henan province, and Qianxinan of Guizhou province (Fig. 5, Table 2). Among these 8 species, Ochrobactrum anthropi from Mangdui and Pantoea agglomerans from Chuxiong have been previously reported as strains capable of heavy metal detoxification. Leclercia adecarboxylata was previously reported as a PAH-degrading strain. Enterobacter ludwigii from Menglijiaojidi is capable of crop-growth promotion and alkane degradation. Ochrobactrum lupini from Qujing was known to degrade pesticides (chlorothalonil and cypermethrin). The other three species identified in the LEfSe have no previously reported functional associations, but their genera do contain species capable of degrading PAHs and heavy metals detoxication.

We explored the potential local factors which have potentially fostered the high relative abundance of these locally specific contaminant-degrading strains, specifically by measuring heavy metal concentrations of ( $\mathrm{Cd}, \mathrm{Cr}, \mathrm{Cu}, \mathrm{Pb}$ and $\mathrm{Zn}$ ) of 6 samples (Mangdui, Menglijiaojidi, Qujing, Chuxiong, Luoyang, and Qianxinan) as well as other 10 samples as references. Compared with other samples (including samples from the same province), Mangdui had the highest the Cd concentration, Chuxiong had the highest $\mathrm{Cr}$ concentration, and Luoyang had the highest $\mathrm{Pb}$ concentration, while Qujing had the second-highest $\mathrm{Cu}$ concentration of any site, and showed significantly higher Zn concentration among the detected samples (Fig. 5). These results are understandable given that Mangdui, Chuxiong, Qujing and Luoyang are areas with abundant complex metal ore and coal mines [50-53]. These LEfSe-based results support that both geographical and industrial factors have substantially driven the microbial community composition in the local environment of the testing sites.

\section{Discussion}

In this study, we systematically examined the national diversity of bacterial communities on leaves of a single crop with 16S rDNA sequencing technology, and found a total of 1,234 species (4,473 OTU) from 78 samples in 8 provinces of China. We determined the core bacterial community of a nation (China) and the core communities for 8 Chinese provinces, which can facilitate future efforts to study crop microbiomes across geographically diverse distributions, which could serve for isolating functional strains on purpose.

It was notable that the South-easterly Fujian province-which has a subtropical monsoon climate characterized by high humidity, high temperature, and abundant precipitation-has the most diverse resources in terms of beneficial bacteria among the examined provinces. Specifically, the Fujian core bacterial core community contained 12 species of beneficial bacteria, a number higher than the other 7 provinces (Table S3). We also noted that Heilongjiang province was apparently the most suitable environment for the beneficial bacteria; this province is in the extreme far North of China and has a cold 
temperate climate in some regions and a temperate continental monsoon climate in others. The abundance of beneficial bacteria in its core community is $39.8 \%$, which is the highest among all of the examined provinces.

Recalling that Bacillus sp. are known to significantly impact the chemical components of tobacco leaves, ${ }^{11}$ it was notable that these taxa occupied more than $1 \%$ of the relative abundance across all samples of the data set. However, no Bacillus sp. were among the national or provincial core bacterial communities. Of the 78 samples, only 16 had more than $1 \%$ relative abundance of Bacillus. These 16 samples were distributed in seven provinces, Yunnan, Fujian, Henan, Heilongjiang, Sichuan, Hunan, and Guizhou. So, although these taxa are distributed all over China, these are clearly not evenly distributed in every region (Figure S5). It also bears mention that Arthrobactersp., which are known to employ the pyridine pathway to degrade nicotine, were among the detected taxa for some samples but were not included among the national or provincial core bacterial communities.

We also performed non-targeted metabolome analysis for the same samples of heavy-metalconcentration measurement (Figure S6). It showed that amino acid synthesis and catabolism, nicotinate and nicotinamide metabolism, and carbapenems synthesis are quite active. Among these, carbapenems synthesis might indicate an unique defense mechanism for native microorganisms on leaves. It might cause low efficiency of the microbial agents applied on tobacco leaves.

\section{Conclusion}

These findings suggest that both climactic and industrial factors drive the microbial community composition in the local environment, and emphasize that there are many potentially useful functional bacterial resources present on tobacco leaves that could be harnessed for a variety of bioprocess, agricultural, and environmental detoxification applications.

\section{Abbreviations}

OTU: Operational taxonomic units; QIIME: Quantitative insights into microbial ecology; SAD: Species abundance distributio; RDA: Redundancy analysis; UPGMA: Unweighted pair-group method with arithmetic mean; NMDS: Non-metric multidimensional scaling; ICP-MS: Inductively coupled plasma mass spectrometry; PICRUSt: Phylogenetic investigation of communities by reconstruction of unobserved states; LEfSe: Linear discriminant analysis effect size; YN: Yunnan province; HeN: Henan province; CQ: Chongqing city; HuN: Hunan; GZ: Guizhou province; SC: Sichuan province; HLJ: Heilongjiang province; FJ: Fujian province. NG: negative group; CX: Chuxiong; LY: Luoyang; MLG: Mengligong; SG: Shigang; XY: Xiangyang; JD: Menglijiaojidi; MD: Mangdui; QJ: Qujing; SMX: Sanmenxia; ZY: Zhuyang; LJ: Lijiang; MDJ: Mudanjiang; QXN: Qianxinan; XC: Xuchang. WS: Wenshan; XD: Xindian; ZW: Zhangwu; SW: Shaowu; SM: Sanming.

\section{Declarations}




\section{Ethics approval and consent to participate}

Not applicable

\section{Consent for publication}

Not applicable

\section{Availability of data and material}

All raw data generated in this study is included in the supplement

Original sequencing datasets are available. Please contact author for data requests.

\section{Competing interests}

The authors declare no competing interests.

\section{Funding}

This study was supported by the grants from Shanghai Excellent Academic Leaders Program (20XD1421900), and from 'Shuguang Program' (17SG09) supported by Shanghai Education Development Foundation and Shanghai Municipal Education Commission.

\section{Author contributions}

Haiyang Hu, Zhan Zhang, and Hongzhi Tang conceived the ideas and designed the experiments. Haiyang Hu, Yunli Liu, Tingting Zhang, Zongcan Yang, Wenzhao Liu, Yingjie Feng and Mingqi Gao performed the experiments. Haiyang Hu, Yunli Liu, and Hongzhi Tang wrote the manuscript. Haiyang Hu, Hongzhi Tang, and Ping Xu revised the manuscript. All Authors commented on the manuscript before submission. All authors read and approved the final manuscript.

\section{Acknowledgments}

We thank Dr. John H. Snyder for assistance and discussion regarding this manuscript.

\section{References}

1. Alivisatos AP, Blaser MJ, Brodie EL, Chun M, Dangl JL, Donohue TJ, et al. A unified initiative to harness Earth's microbiomes. Science. 2015;350:507-8.

2. Fierer N, Jackson RB. The diversity and biogeography of soil bacterial communities. Proc Natl Acad Sci USA. 2006;103:626-31.

3. Bahram M, Hildebrand F, Forslund SK, Anderson JL, Soudzilovskaia NA, Bodegom PM, et al. Structure and function of the global topsoil microbiome. Nature. 2018;560:233-7. 
4. Löffler FE, Edwards EA. Harnessing microbial activities for environmental cleanup. Curr Opin Biotech. 2006;17:274-84.

5. Wu L, Ning D, Zhang B, Li Y, Zhang P, Shan X, et al. Global diversity and biogeography of bacterial communities in wastewater treatment plants. Nat Microbiol. 2019;4:1183-95.

6. Delgado-Baquerizo M, Oliverio AM, Brewer TE, Benavent-González A, Eldridge DJ, Bardgett RD, et al. A global atlas of the dominant bacteria found in soil. Science. 2018;359:320-5.

7. Ladha, JK, Barraquio, WL, Watanabe, I. Isolation and identification of nitrogen-fixing Enterobacter cloacae and Klebsiella planticola associated with rice plants. Can J Microbiol. 1983;29:1301-8.

8. Ritpitakphong U, Falquet L, Vimoltust A, Berger A, Métraux JP, L'Haridon F. The microbiome of the leaf surface of Arabidopsis protects against a fungal pathogen. New Phytol. 2016;210:1033-43.

9. Busby PE, Peay KG, Newcombe G. Common foliar fungi of Populus trichocarpa modify Melampsora rust disease severity. New Phytol. 2015;209:1681-92.

10. Leveau JH. A brief from the leaf: latest research to inform our understanding of the phyllosphere microbiome. Curr Opin Microbiol. 2019;49:41-9.

11. English CF, Bell EJ, Berger AJ. Isolation of thermophiles from broadleaf tobacco and effect of pure culture inoculation on cigar aroma and mildness. Appl Microbiol. 1967;15:117-9.

12. Ruiz Gutierrez V. Nicotine degradation by bacteria Enterobacter cloacae as nicotine degrader. Tobacco. 1983;22:85-98.

13. Zhao M, Cui J. Analysis of bacterial communities on aging flue-cured tobacco leaves by $16 \mathrm{~S}$ rDNA PCR-DGGE technology. Appl Microbiol Biotechnol. 2007;73:1435-40.

14. Zhao M, Liu Y, Li F, Wang B, Liu G. Identification of dominant and fragrance-enhancing microorganisms of tobacco leaves during ripening. Acta Microbiologica Sinica. 2009;49:624-630.

15. Huang J, Yang J, Duan Y, Gu W, Gong X, Zhe W, et al. Bacterial diversities on unaged and aging fluecured tobacco leaves estimated by $16 \mathrm{~S}$ rRNA sequence analysis. Appl Microbiol Biotechnol. 2010;88:553-62.

16. Su C, Gu W, Zhe W, Zhang KQ, Duan Y, Yang J, et al. Diversity and phylogeny of bacteria on Zimbabwe tobacco leaves estimated by $16 \mathrm{~S}$ rRNA sequence analysis. Appl Microbiol Biotechnol. 2011;92:1033-44.

17. Wang Y, Xie XR, Lei LP, Xia ZY. The diversity analysis of bacteria in curing tobacco leaves. Chinese Journal of Biological Control. 2009:15:20-4.

18. Chen ZT, Yan TT, Tang CQ, Yong-Sheng QU, Peng-Fu LI, et al. Analyzing bacterial diversity in aging flue-cured tobacco leaves using 16S rDNA clone library analysis. Acta Tabacaria Sinica. 2012;18:7782.

19. Xue-Ying WU, Liang SL, Han SY, Chen XM, Lin Y, et al. Diversity and phylogenetic analysis of bacterial communities on flue-cured tobacco leaves at different aged phases. Guangdong Agricultural Sciences. 2014;9:28-33. 
20. Shannon CE. A mathematical theory of communication. Bell Labs Technical Journal. 1948;27:379423.

21. Simpson EH. Measurement of diversity. Nature. 1949;163:261.

22. Chao A, Chazdon RL, Colwell RK, Shen TJ, et al. A new statistical approach for assessing similarity of species composition with incidence and abundance data. Ecol Lett. 2010;8:148-59.

23. Chao A, Yang M. Stopping rules and estimation for recapture debugging with unequal failure rates. Biometrika. 1993;80:193-201.

24. McGill BJ, Etienne RS, Gray JS, Alonso D, Anderson MJ, Benecha HK, et al. Species abundance distributions: moving beyond single prediction theories to integration within an ecological framework. Ecol Lett. 2007;10:995-1015.

25. Langille MG, Zaneveld J, Caporaso JG, McDonald D, Knights D, Reyes JA, et al. Predictive functional profiling of microbial communities using 16S rRNA marker gene sequences. Nat Biotechnol. 2013;31:814-21.

26. Saunders AM, Albertsen M, Vollertsen J, Nielsen PH. The activated sludge ecosystem contains a core community of abundant organisms. ISME J. 2016;10:11-20.

27. Gibson DJ, Ely JS, Collins SL. The core-satellite species hypothesis provides a theoretical basis for Grime's classification of dominant, subordinate, and transient species. J Ecol. 1999;87:1064-7.

28. Guida BS, Garcia-Pichel F. Draft genome assembly of a filamentous euendolithic (true boring) cyanobacterium, Mastigocoleus testarum strain BC008. Genome Announc. 2016;4:e01574-15.

29. Hata H, Natori T, Mizuno T, Kanazawa I, Eldesouky I, Hayashi M, et al. Phylogenetics of family Enterobacteriaceae and proposal to reclassify Escherichia hermannii and Salmonella subterranea as Atlantibacter hermannii and Atlantibacter subterranea gen. nov., comb. nov. Microbiol Immunol. 2016;60:303-11.

30. Feng YJ, Shen DL, Song W. Rice endophyte Pantoea agglomerans YS19 promotes host plant growth and affects allocations of host photosynthates. J Appl Microbiol. 2010;100:938-45.

31. Francis CA, Obraztsova AY, Tebo BM. Dissimilatory metal reduction by the facultative anaerobe Pantoea agglomerans SP1. Appl Environ Microbiol. 2000;66:543-8.

32. Nunes $\mathrm{C}$, Usall J, Teixidó $\mathrm{N}$, Viñas I. Biological control of postharvest pear diseases using a bacterium, Pantoea agglomerans CPA-2. Int J Food Microbiol. 2001;70:53-61.

33. Vagelas IK, Pembroke B, Gowen SR. The control of root-knot nematodes (Meloidogyne spp.) by Pseudomonas oryzihabitans and its immunological detection on tomato roots. Nematology. 2007;9:363-70.

34. Sarma PM, Duraja P, Deshpande S, Lal B. Degradation of pyrene by an enteric bacterium, Leclercia adecarboxylata PS4040. Biodegradation. 2010;21:59-69.

35. Daniel KM, William JH, Jorge MV. Enterobacter soli sp. nov.: A lignin-degrading y-proteobacteria isolated from soil. Curr Microbiol. 2011;62:1044-9. 
36. Greenhut IV. iac gene expression in the indole-3-acetic acid-degrading soil bacterium Enterobacter soli LF7. Appl Environ Microbiol. 2018;84:e01057-18.

37. Wei $\mathrm{CH}$, Ren Y, Wu CF. The characteristics of aniline biodegradation by Ochrobactrum anthropi. Chinese Journal of Environmental Science. 1998;19:22-4.

38. Ozdemir G, Ozturk T, Ceyhan N, Isler R, Cosar T. Heavy metal biosorption by biomass of Ochrobactrum anthropi producing exopolysaccharide in activated sludge. Bioresour Technol. 2003;90:71-4.

39. Shi XZ, Guo RJ, Takagi K, Miao ZQ, Li SD. Chlorothalonil degradation by Ochrobactrum lupini strain TP-D1 and identification of its metabolites. World J Microb Biot. 2011;27:1755-64.

40. Chen S, Hu M, Liu J, Zhong G, Yang L, Rizwan-ul-Haq M, et al. Biodegradation of beta-cypermethrin and 3-phenoxybenzoic acid by a novel Ochrobactrum lupini DG-S-01. J Hazard Mater. 2011;187:433-40.

41. Bhattacharya M, Biswas D, Sana S, Datta S. Utilization of waste engine oil by Ochrobactrum pseudintermedium strain $\mathrm{C} 1$ that secretes an exopolysaccharide as a bioemulsifier. Biocatal Agric Biotechnol. 2014;3:167-76.

42. Ontañon OM, González PS, Agostini E. Biochemical and molecular mechanisms involved in simultaneous phenol and $\mathrm{Cr}(\mathrm{VI})$ removal by Acinetobacter guillouiae SFC 500-1A. Environ Sci Pollut Res Int. 2015;22:13014-23.

43. Wang HB, Han LR, Feng JT, Zhang X. Screening of highly efficient cellulose Degradation Microbes and Construction of Composite Strains. Journal of Agricultural Biotechnology. 2015;23:421-31.

44. Zeng XM, Huang QZ. Identification on the pathogenic bacteria of a new tabacco disease-black rot. J South China Agr Univ. 1994;15:46-9.

45. Thele R, Gumpert $H$, Christensen LB, Worning P, Schønning K, Westh $H$, et al. Draft genome sequence of a Kluyvera intermedia isolate from a patient with a pancreatic abscess. J Glob Antimicrob Resist. 2017;10:1-2.

46. Nicodemo AC, Paez JI. Antimicrobial therapy for Stenotrophomonas maltophilia infections. Eur J Clin Microbiol Infect Dis. 2007;26:229-37.

47. Zelazny AM, Ding L, Goldberg JB, Mijares LA, Conlan S, Conville PS, et al. Adaptability and persistence of the emerging pathogen Bordetella petrii. PLoS One. 2013;8:e65102.

48. Kattar MM, Chavez JF, Limaye AP, Rassoulian-Barrett SL, Yarfitz SL, Carlson LC, et al. Application of $16 \mathrm{~S}$ rRNA gene sequencing to identify Bordetella hinzii as the causative agent of fatal septicemia. $J$ Clin Microbiol. 2000;38:789-94.

49. Fuhrman JA, Steele JA, Hewson I, Schwalbach MS, Brown MV, Green JL, et al. A latitudinal diversity gradient in planktonic marine bacteria. Proc Natl Acad Sci USA. 2008;105:7774-8.

50. Gao ZQ, Luo ZX, Meng FJ, Tan X. An analysis of prospecting potentiality of haoziba Pb-Zn multimetallic deposit in Yongde, Yunnan. Yunnan Geology. 2019;38:311-5. 
51. Han RS, Wu P, Hu YZ, Wu HZ. Metallogenic structural plane of lithology and lithofacies in sandstonetype copper deposits in the Chuxiong basin. Advances in Earth Sciences. 2012;S1:174-8.

52. Zheng DF. Metallogenic control factors and ore prospecting significance of Luoyang lead-zinc deposit. World nonferrous metals. 2017;24:110-1.

53. Lu ZJ. The feature and ore control factor of Fusheng Pb-Zn deposit in Fuyuan, Yunnan. Yunnan Geology. 2019;38:426-30.

\section{Tables}

Table 1. The list of the national core microbial community.

\begin{tabular}{llll} 
No. & Genus & Specy & Function \\
\hline 1 & Mastigocoleus & $\begin{array}{l}\text { Mastigocoleus } \\
\text { testarum }\end{array}$ & Function unknown \\
\hline 2 & Atlantibacter & Atlantibacter hermannii & Function unknown \\
\hline 3 & Salmonella & Salmonella enterica & Harmful for human body \\
\hline 4 & Leclercia & $\begin{array}{l}\text { Leclercia } \\
\text { adecarboxylata }\end{array}$ & Degradation of PAHs (pyrene) \\
\hline 5 & Enterobacter & Enterobacter soli & Degradation of indole-3-acetic acid and lignin \\
\hline 6 & Pantoea & $\begin{array}{l}\text { Pantoea agglomerans } \\
\text { heavy metals reduction, nitrogen fixation, }\end{array}$ \\
\hline 7 & Pseudomonas & $\begin{array}{l}\text { Pseudomonas } \\
\text { oryzihabitans } \\
\text { insecticidal activity }\end{array}$ & Insecticidal activity (root-knot nematode) \\
\hline 8 & Methylobacterium & $\begin{array}{l}\text { Methylobacterium } \\
\text { goesingense }\end{array}$ & Function unknown \\
\hline 9 & Sphingomonas & $\begin{array}{l}\text { Sphingomonas } \\
\text { roseiflava } \\
\text { Sphingomonas } \\
\text { aurantiaca }\end{array}$ & Function unknown \\
\hline
\end{tabular}

Table 2. The significantly different species among the samples. 


\begin{tabular}{lll}
$\begin{array}{l}\text { Sampling } \\
\text { location }\end{array}$ & Specy & Function \\
\hline $\begin{array}{l}\text { Yunnan- } \\
\text { Mangdui }\end{array}$ & $\begin{array}{l}\text { Ochrobactrum } \\
\text { anthropi }\end{array}$ & Adsorption of heavy metal ions \\
\hline $\begin{array}{l}\text { Yunnan- } \\
\text { Menglijiaojidi }\end{array}$ & $\begin{array}{l}\text { Leclercia } \\
\text { adecarboxylata }\end{array}$ & Degradation of PAHs \\
\hline $\begin{array}{l}\text { Yunnan- } \\
\text { Menglijiaojidi }\end{array}$ & $\begin{array}{l}\text { Enterobacter } \\
\text { ludwigii }\end{array}$ & Promotion of plant growth, Alkane degradation \\
\hline $\begin{array}{l}\text { Yunnan- } \\
\text { Qujing }\end{array}$ & $\begin{array}{l}\text { Ochrobactrum } \\
\text { lupini }\end{array}$ & Degradation of pesticides (chlorothalonil and cypermethrin) \\
\hline $\begin{array}{l}\text { Yunnan- } \\
\text { Chuxiong }\end{array}$ & $\begin{array}{l}\text { Pantoea } \\
\text { agglomerans }\end{array}$ & Heavy metals detoxification \\
\hline $\begin{array}{l}\text { Henan- } \\
\text { Xuchang }\end{array}$ & $\begin{array}{l}\text { Enterobacter } \\
\text { xiangfangensis }\end{array}$ & $\begin{array}{l}\text { Function unknown, but the corresponding genus is able to } \\
\text { degrade PAHs and heavy metals }\end{array}$ \\
\hline $\begin{array}{l}\text { Henan- } \\
\text { Luoyang }\end{array}$ & $\begin{array}{l}\text { Methylobacterium } \\
\text { brachiatum }\end{array}$ & $\begin{array}{l}\text { Function unknown, but the corresponding genus is able to } \\
\text { degrade PAHs and heavy metals }\end{array}$ \\
\hline $\begin{array}{l}\text { Guizhou- } \\
\text { Qianxinan }\end{array}$ & $\begin{array}{l}\text { Sphingomonas } \\
\text { phyllosphaerae }\end{array}$ & $\begin{array}{l}\text { Function unknown, but the corresponding genus is able to } \\
\text { degrade PAHs and heavy metals }\end{array}$ \\
\hline
\end{tabular}

\section{Figures}


a

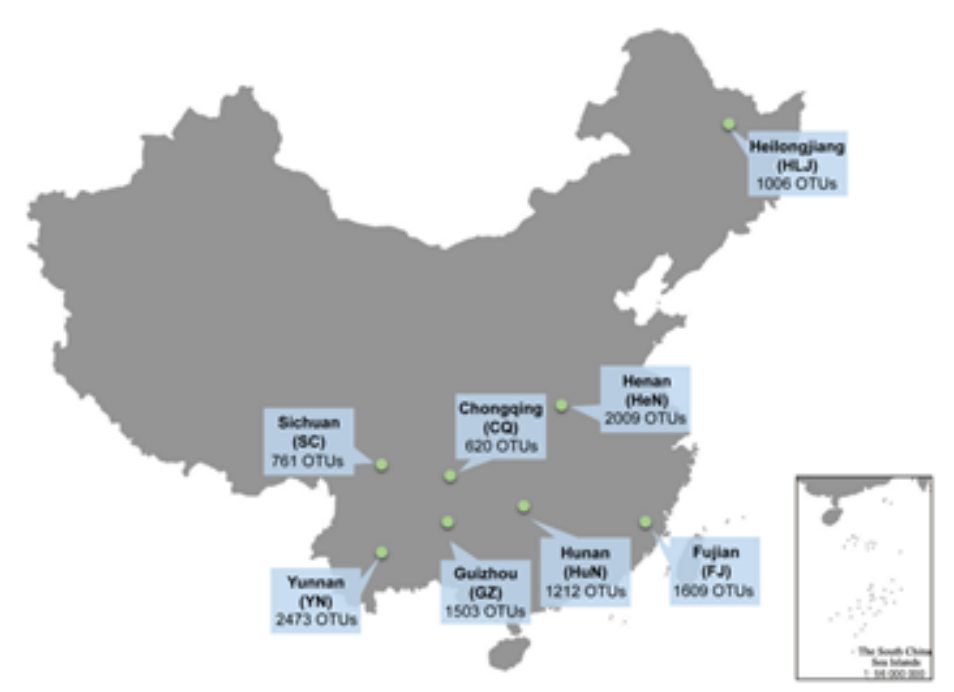

b

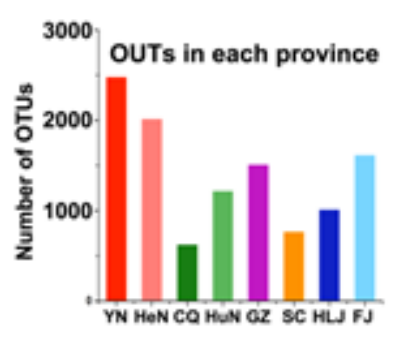

C

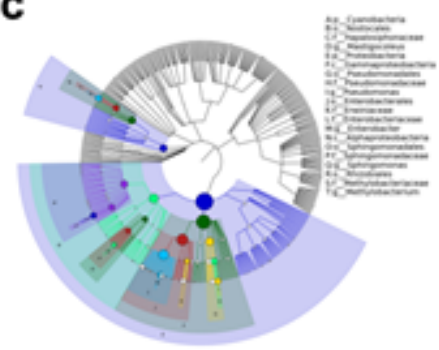

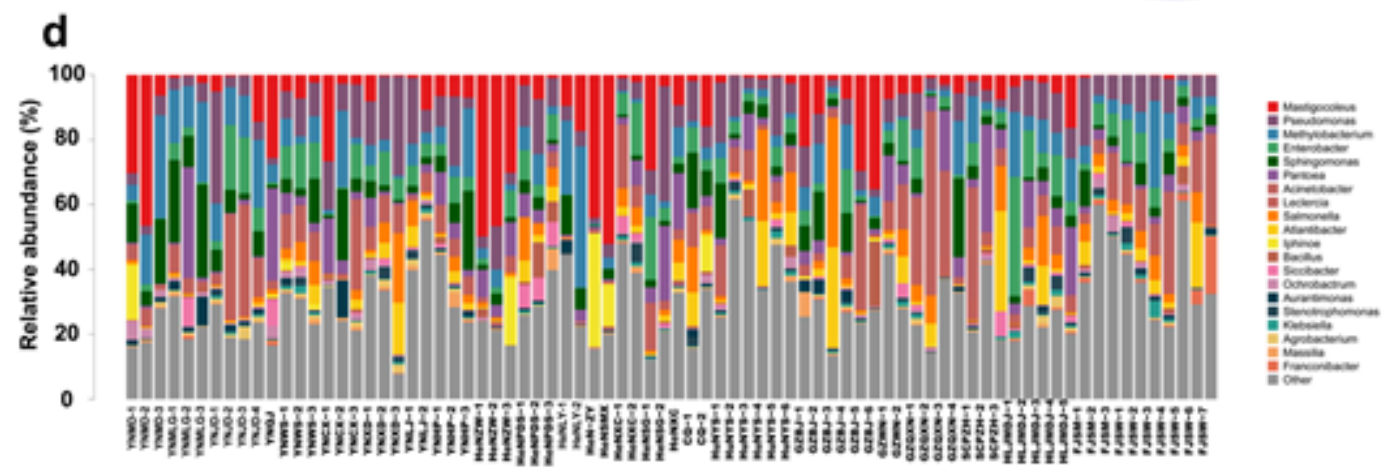

\section{Figure 1}

Sampling and initial phylogenic distributions. A Sampling locations of the present study. B Number of OTUs in each province. YN, Yunnan province; HeN, Henan province; CQ, Chongqing city; HuN, Hunan; GZ, Guizhou province; SC, Sichuan province; HLJ, Heilongjiang province; FJ, Fujian province. C Classification tree for all samples, visualized using GraPhIAn. D The community compositions at the genus-level. The relative abundances of the following genera were $>1 \%$ : Mastigocoleus, Pseudomonas, Methylobacter, Enterobacter, Sphingomonas, Pantoea, Acinetobacter, Leclercia, Salmonella, Atlantibacter, Iphinoe, Bacillus, and Siccibacter. 
a

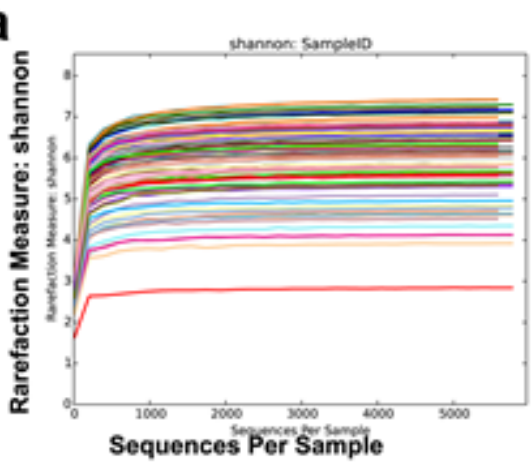

C

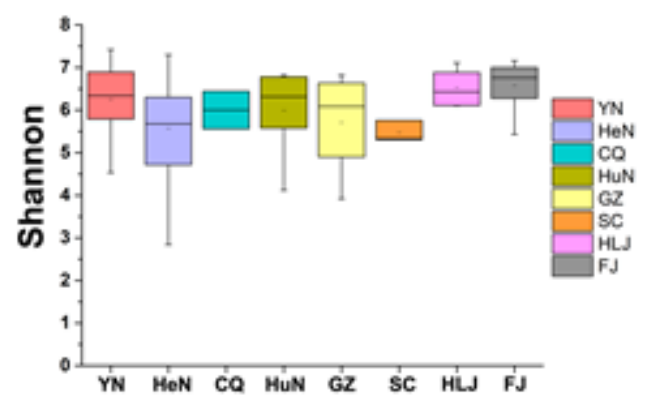

b

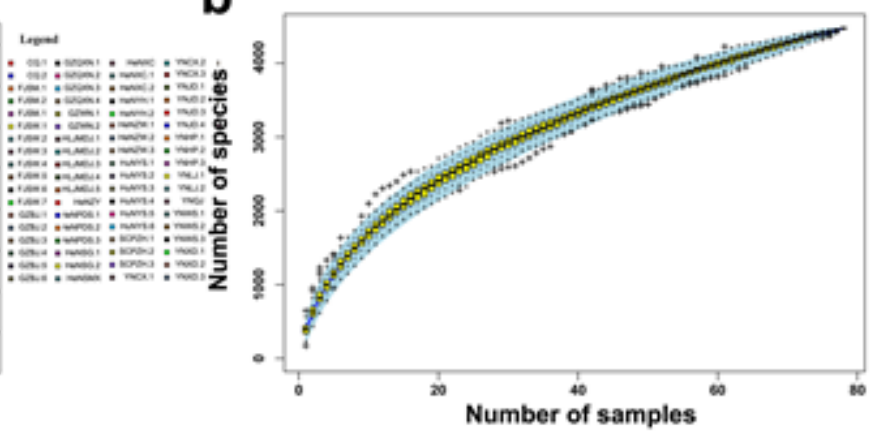

d

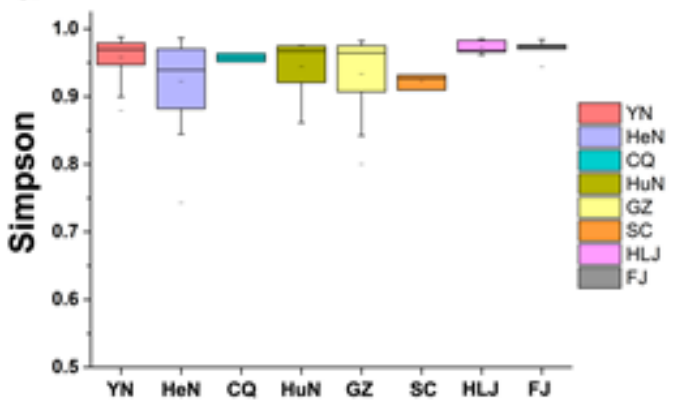

Figure 2

a-diversity analysis. A The Shannon rarefaction curves of 78 samples. The sequencing depth of this study is adequate to reveal the community diversity of the samples. B Species accumulation curves of 78 samples. The bacterial richness of the 78 samples is sufficient for further analysis. C Box plots of the Shannon index values. D Box plots of the Simpson index values. Microbial diversity was highest in Fujian province and lowest in Henan province. 


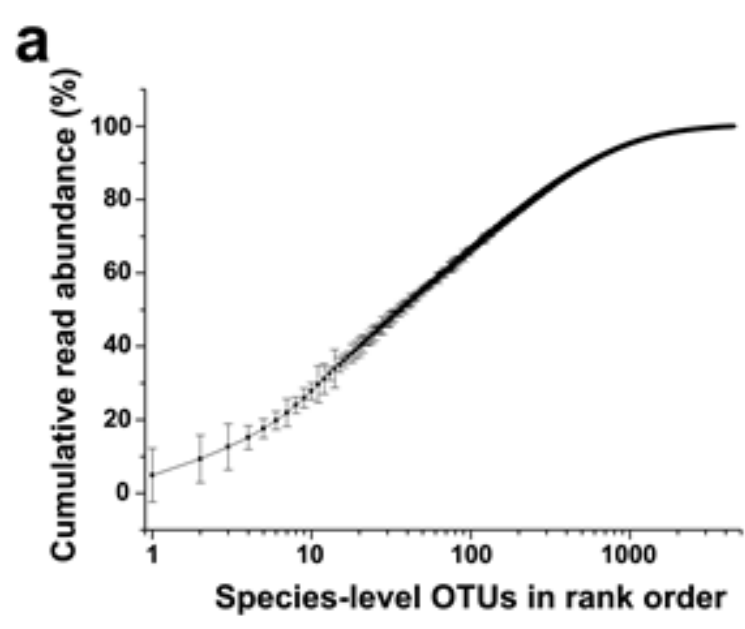

C

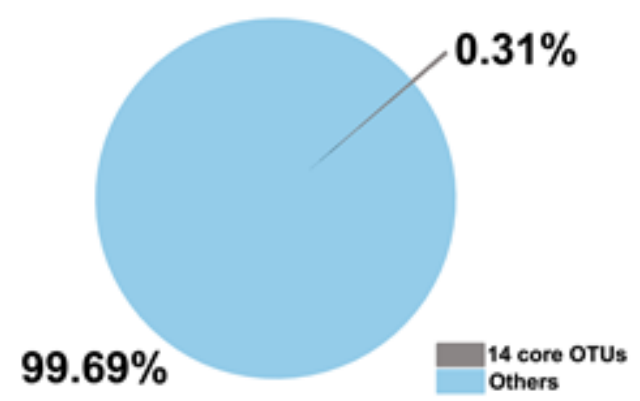

Number of OTUs
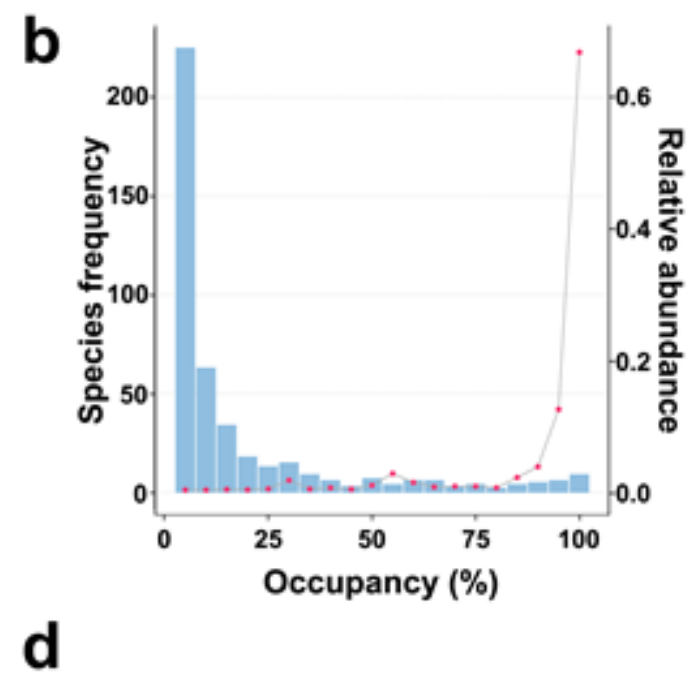

d

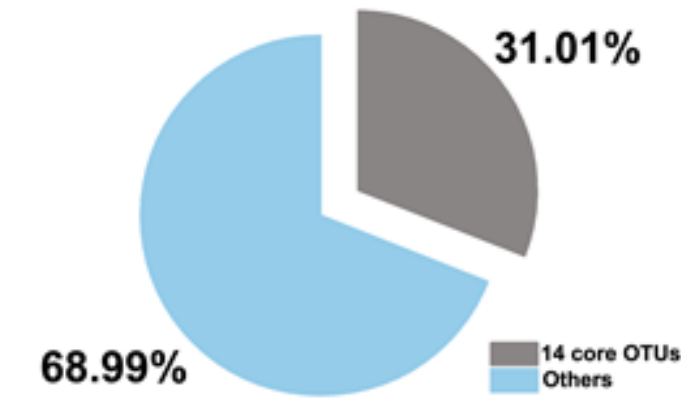

Relative abundance

\section{Figure 3}

Core bacterial community. A Cumulative read abundance across the 78 samples. Cumulative read abundance (mean \pm SD) of species-level OTUs plotted in rank order for the 78 samples. In each sample, the 10 most abundant OTUs made up $27.78 \%( \pm 2.26 \%)$ of the total reads on average, and the 100 most abundant OTUs made up $66.32 \%$ ( $\pm 0.34 \%)$. OTUs were considered "abundant" in a sample when they were among the OTUs comprising the top $80 \%$ of reads. B Frequency and abundance distributions of all OTUs. OTUs were classified into bins according to their occupancy of samples. For example, the first bin contains OTUs detected in fewever than $5 \%$ of the samples, while the last bin contains OTUs detected in $95 \%-100 \%$ of the samples. Species frequency (the number of OTUs) is indicated by the blue bars; the total abundance of OTUs in each bin is indicated by the red points. C, D Percentage and relative abundance of the core OTUs versus the remaining microbial OTUs. In total, $0.31 \%$ ( 14 of 4,473 OTUs) OTUs were identified as abundant and frequent at the countrywide scale; these OTUs accounted for (on average) $31.01 \%$ of the $16 \mathrm{~S}$ rRNA gene sequences of all samples. 



Figure 4

$\beta$-diversity and redundancy analysis. A Multi Response Permutation Procedure (MRPP). The composition difference between each province was valued by $-\log 10(P)$. The red dots are the pairs that differ significantly, while the blue dots are that do not differ. More than half of the pairs showed significant differences, indicating that the microbial community composition varies greatly from province to province. B Redundancy analysis. The relationship between microbial diversity and temperature, precipitation, altitude, longitude, and latitude of each sample was analyzed. Each arrow represents an environmental factor, with each dot representing one sample. An acute angle between arrows indicates a positive correlation, an obtuse angle indicates a negative correlation, and a right angle indicates no correlation. 

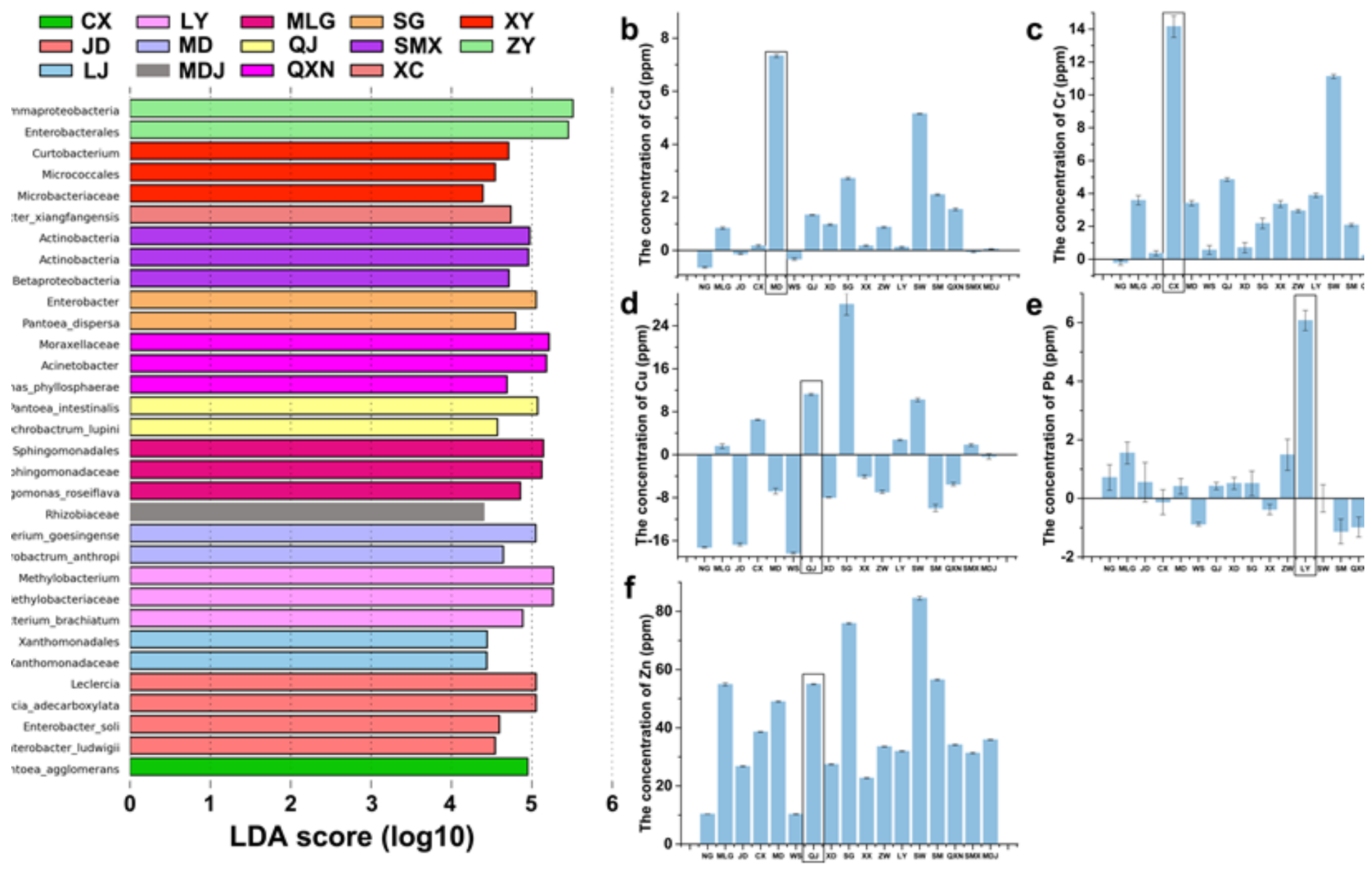

\section{Figure 5}

LEfSe analysis and heavy metal concentration determination. A LEfSe (Linear discriminant analysis effect size). The ordinate is the taxon which differs significantly between groups; the abscissa visually shows the LDA difference analysis logarithm score ( $L D A>2, P<0.05)$ for the corresponding taxon (as a bar graph). B The concentration of Cd. C The concentration of Cr. D The concentration of Cu. E The concentration of $\mathrm{Pb}$. $\mathrm{F}$ The concentration of $\mathrm{Zn}$. Negative values refer to no ions present. NG, negative group; CX, Chuxiong; LY, Luoyang; MLG, Mengligong; SG, Shigang; XY, Xiangyang; JD, Menglijiaojidi; MD, Mangdui; QJ, Qujing; SMX, Sanmenxia; ZY, Zhuyang; LJ, Lijiang; MDJ, Mudanjiang; QXN, Qianxinan; XC, Xuchang. WS, Wenshan; XD, Xindian; ZW, Zhangwu; SW, Shaowu; SM, Sanming.

\section{Supplementary Files}

This is a list of supplementary files associated with this preprint. Click to download.

- SupplementaryInformationEnvironmentalMicrobiome.doc 